\title{
Semiconductor to Metal Transition in Ferric Molybdate
}

\author{
K. TENNAKONE AND W. G. D. DHARMARATNA \\ Ruhuna University College, Matara, Sri Lanka.
}

(Date of receipt: 27 September 1982)

(Date of acceptance: 06 July 1983)

\begin{abstract}
Conductivity characteristics of ferric molybdatc is studied. It is found that this material undergoes a semiconductor to metal transition at $\sim 139^{\circ} \mathrm{C}$.
\end{abstract}

\section{Introduction}

The study of insulator to metal and semiconductor to metal transitions in inorganic solids has attracted much attention. ${ }^{2,8}$ It is well known that transition metal compounds, notably those of iron exhibits this phenomenon at relatively low temperatures. ${ }^{2.4 .7}$ In this note we describe our observations on semiconductor to metal transition in ferric molybdate $\left(\mathrm{Fe}_{2}\left[\mathrm{MoO}_{4}\right]_{3}\right)$.

\section{Method}

To obtain reproducible results, ferric molybdate is prepared by the following methods. Dilute acetic acid (10\% solution) is added to $0.25 \mathrm{M}$ ammonium molybdate in ammonium hydroxide ( contaning $2 \% \mathrm{NH}_{3}$ ) until the $\mathrm{pH}$ is $\bumpeq 5$. Excess ferric chloride solution containing acetic acid $(\mathrm{pH}$.is $\bumpeq 4.5$ ) is added dropwise with vigorous stirring to the molybdate solution and the mixture is boiled. The yellowish brown precipitate of ferric molybdate is washed with deionized water and dried for several hours at $200^{\circ} \mathrm{C}$. The dried compound chocolate brown in colour is powdered and compacted between carbon electrodes at a pressure of $\sim 5 \times 10^{5} \mathrm{~Pa}$ in a pyrex glass tube (diameter $\bumpeq 0.5 \mathrm{~cm}$, pellet length $\bumpeq 0.4 \mathrm{~cm}$.). At this pressure the effect of grain boundaries is found to be negligible and further increase of pressure does not reduce the conductivity significantly. The ends of the tube are sealed with epoxy resin, the sample is immersed in a thermostatic bath and V-I characteristics are determined at various temperatures.

\section{Results and Discussion}

The voltage - current characteristics are found to be highly nonlinear. The plots of current density $(\mathrm{J})$ vs potential gradient $(\mathrm{E})$ at different temperatures are indicated in Figure I. The transition to the highly conducting metallic phase is dependent upon temperature as well as current density. At a given temperature less than $T_{\mathrm{C}}\left(\bumpeq 139^{\circ} \mathrm{C}\right)$, as the voltage is increased from zero, J varies continuously until a point on the curve $A$ is reached. Once this point is passed, there is an abrupt increase in conductivity and 


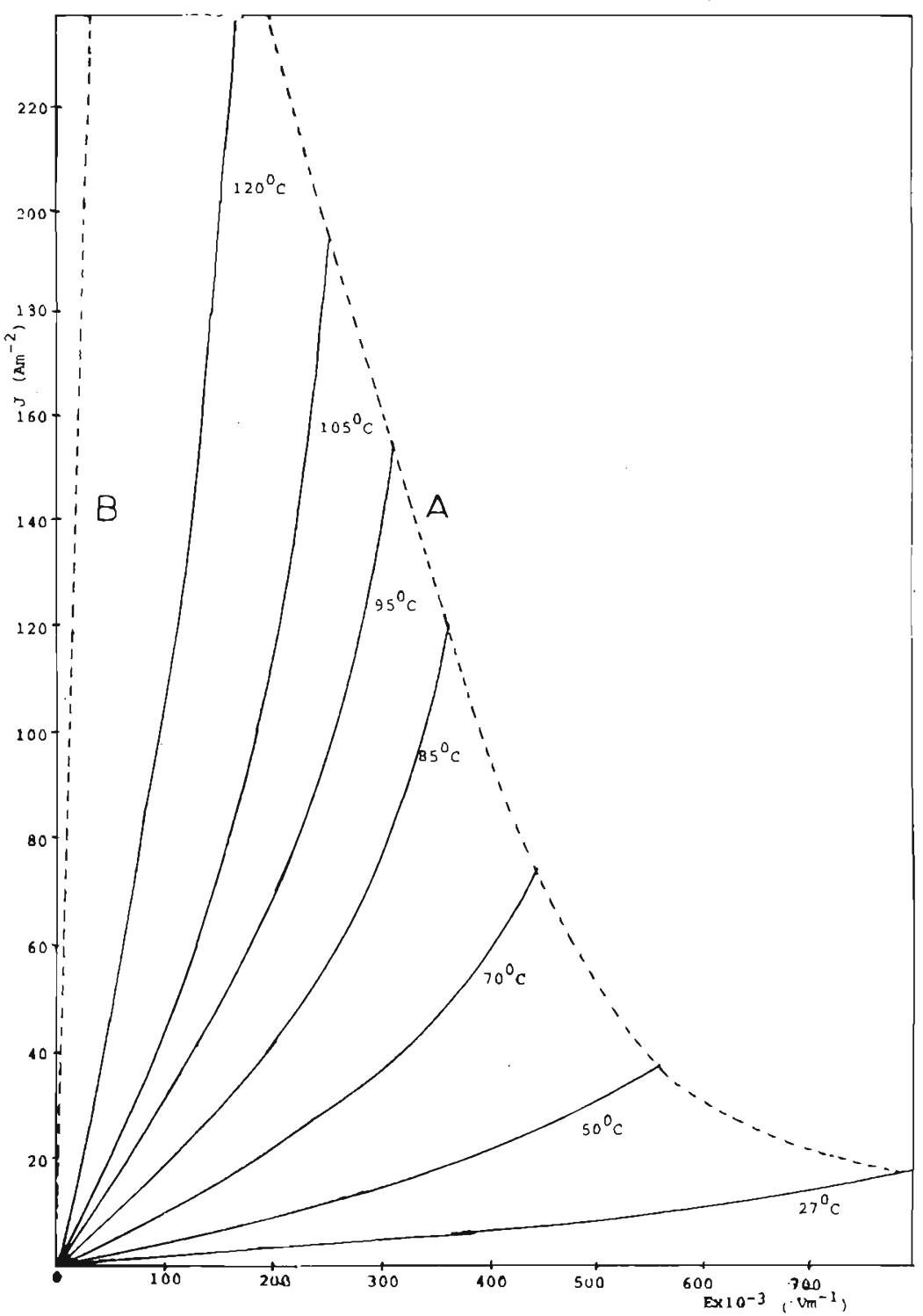

Fig. 1. The plot of $\mathbf{J}$ vs $\mathrm{E}$ at different temperatures. The dashed curve $\mathrm{A}$ gives the points at which the transition to metallic phase takes place abruptly when $J$ is increased. When $T>T_{c}\left(=139^{\circ} \mathrm{C}\right)$ the material transforms into the conducting phase and $J, E$ vary along the line. $B$. 
further increase of $\mathrm{E}$ will change $\mathrm{J}$ along the line $\mathrm{B}$, where the conductivity is almost constant ( $\simeq 4.5 \times 10^{2} \mathrm{sm}$ ). No hysteresis is observed before the curve $A$ is intersected, i.e. if the voltage is decreased before crossing this curve, $J$ decreases along the initial path via which it had increased. When the point where the abrupt increase in conductivity is passed, the material remains in the highly conducting metastable phase for several minutes even if the current is discontinued.

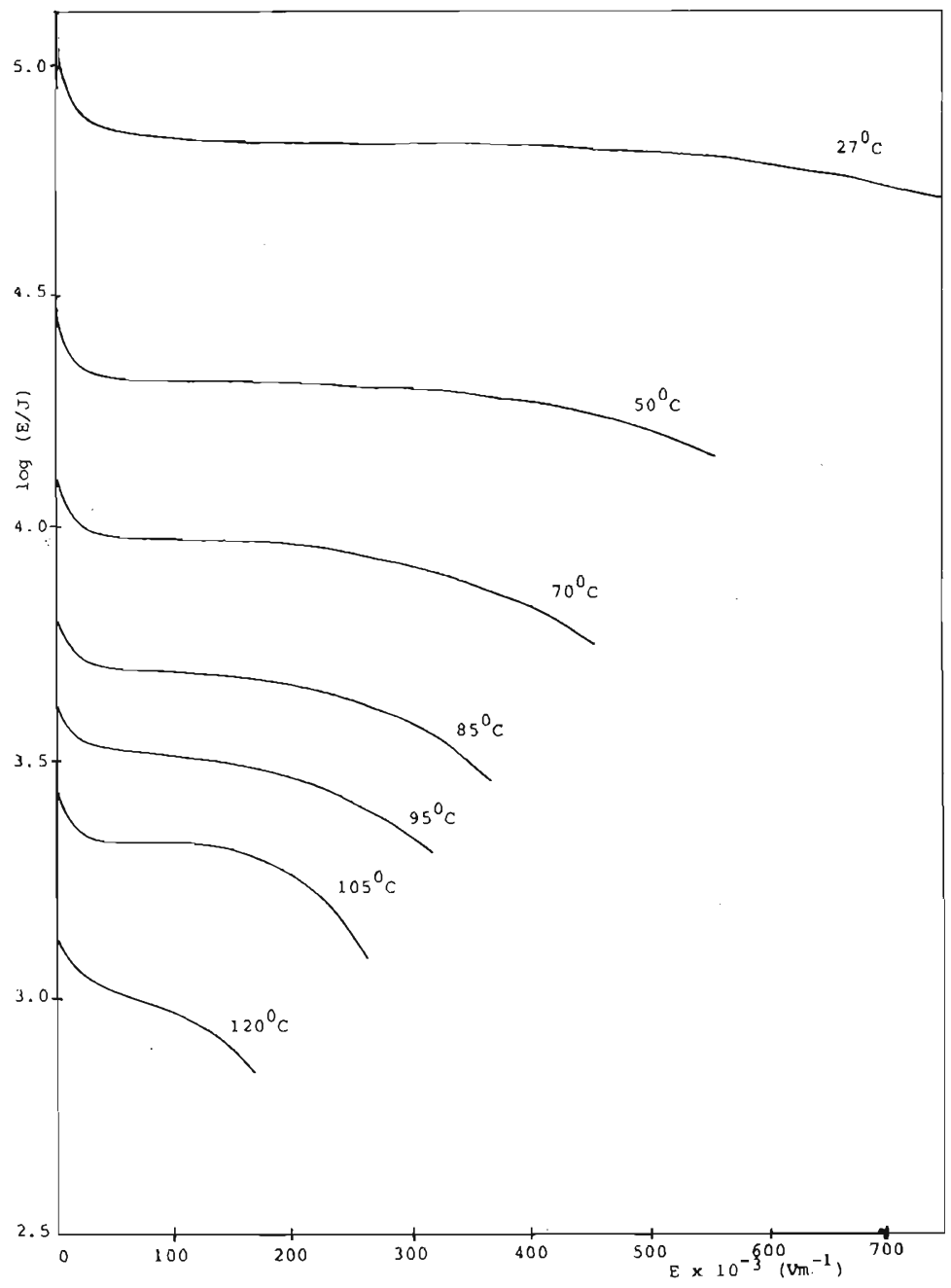

Fig. 2. Plot of $\log (E / J)$ vs $E$ at different temperatures. 


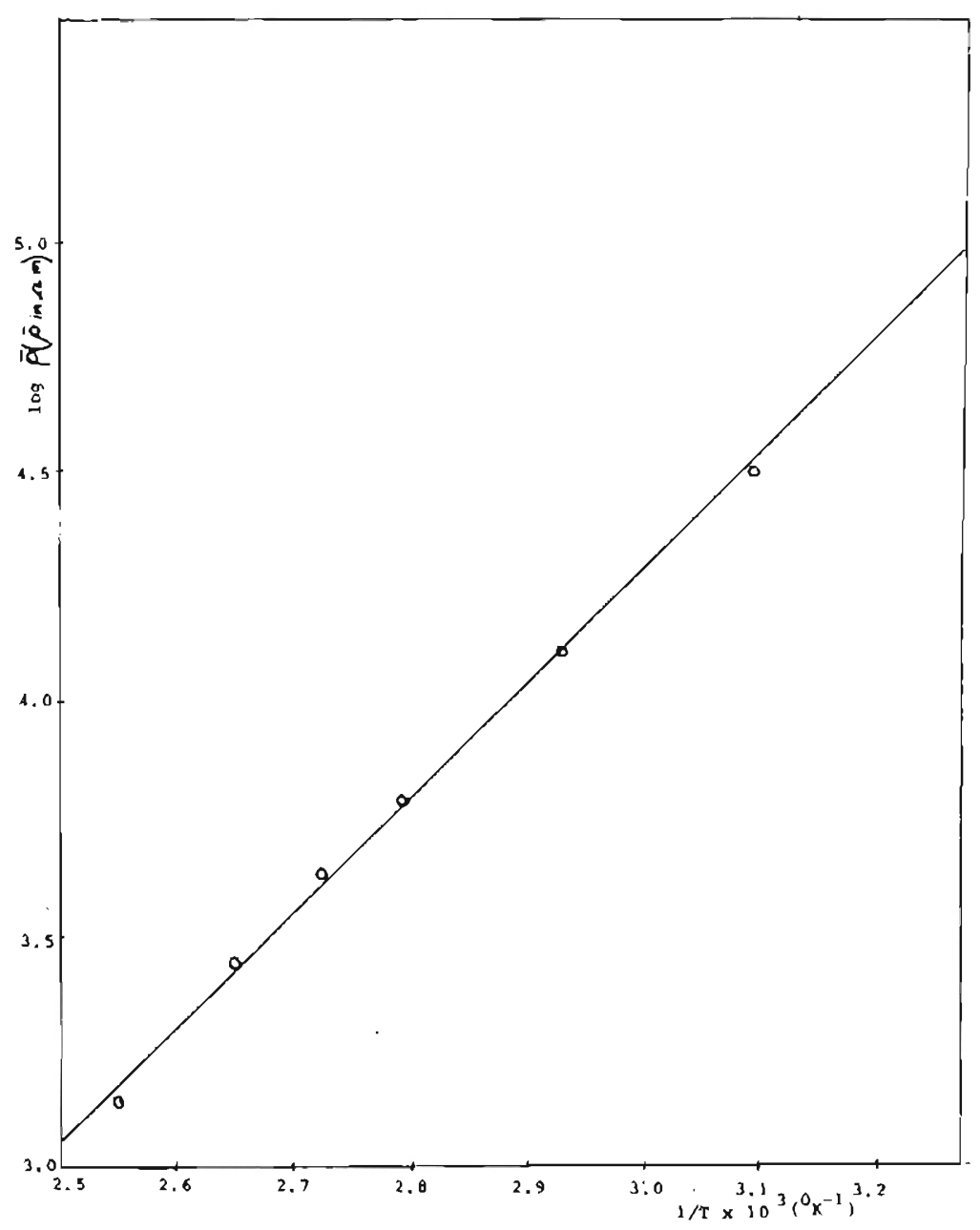

Fig. 3. Plot of $\log \bar{\rho}$ vs $T^{-1}$ in the semiconducting phase. $\{\bar{\rho}=$ resistivity at zero current density).

Again if the critical temperature $T_{C} \bumpeq 139^{\circ} \mathrm{C}$ is exceeded, the material transforms into the metallic phase, i.e. the current voltage characteristics follow the path $\mathrm{B}$ for all current densities including the zero limit (Figure 1). Also if the sample is cooled once the temperature had exceeded $T_{C}$, material remains in the metallic phase and complete reversal to the semiconducting phase is observed after several hours.

From a plot of $\log (E / J)$ vs $E$ (Figure 2 ) it is possible to determine the resistivity $\bar{\rho}$ of the material at different temperatures as the limit $E \rightarrow 0, J \rightarrow 0$ is approached in the semiconducting phase. The plot of $\log \bar{\rho}$ vs $\mathrm{T}_{-1}$ is a straight line (Figure 3 ) indicat ing that the relation, 


$$
\bar{\rho}=\bar{\rho}_{0} \mathrm{e}^{\mathrm{E} / \mathrm{kT}}
$$

is satisfied with $\hat{\rho}_{n}=6.3 \times 10-4 \mathrm{~nm}$ and activation energy $\mathrm{E}=0.50 \mathrm{eV} .\left(\hat{\rho}\right.$ at $139^{\circ} \mathrm{C}$ computed from (1) is $\sim 7.1 \times 10^{2} \Omega \mathrm{m}$, thus there is $\sim 10^{4}$ fold increase in conductivity after the transition).

The system is highly sensitive to impurities. The samples of the compound prepared in the manner described above corresponds to stoichimetric $\mathrm{Fe}_{2}\left(\mathrm{MoO}_{4}\right)_{3}$ to an accuracy of $\pm 0.3 \%$ in the content of $\mathrm{Fe}$ and $\mathrm{O}$. Although reproducible results are obtained, the presence of $\mathrm{Fe}_{2} \mathrm{O}_{3}$ as an impurity in the samples cannot be ruled out. If a neutral instead of acidic solution of ammonium molybdate is used in the preparation, the resulting material has reproducible but slightly different conductivity properties; i.e. the transition temperature is increased by several degrees and the conductivity in the metallic phase is reduced.

There is no evidence for ionic conductivity ${ }^{3.5}$, however we have not been able to determine the electron and/or hole mobilities in the material. The crystal structure of ferric molybdate is not reported in literature to the knowledge of the authors.

\section{References}

1. AUSTIN, I. G. \& MOTT, N. F. (1970). Science 168, 71

2. HENISCH, H. K. (1952), Semiconducting Materials Butterworths, London

3. HLADIC, J. (1972). Physics of Electrolytes: ed. by J. Hladic Academic New York.

4. KLEIN, N., I'ANNAÚSER, D. S. \& HOLLAK, B. \& N. (1971). Conduction in Low-Mobility Materials, Proceedings 2nd International Conference, eds.

5. MAHAN, G. D. \& ROTH, W. L. (1976). Superionic Conduciors Plenum, New York.

6. MOTT, N. F. (1956). Canadian Journal of Physics 34: 1356.

7. MOTT, N. F. (1961). Phil. Mag. 6: 287.

8. MOTT, N. F. \& DAVIS, E. A. (1971). Electronic Processes in Non-Crystalline Solids Oxford University Press, London. 